\title{
LOS FUNDAMENTOS EPISTEMOLÓGICOS DE LA TRANSFORMACIÓN DIGITAL Y SUS EFECTOS SOBRE LA AGENDA 2030 Y LOS DERECHOS HUMANOS ${ }^{1}$
}

\author{
Autor: Joaquín Fernández Mateo \\ joaquin.fernandez@urjc.es \\ Universidad Rey Juan Carlos
}

\begin{abstract}
Resumen
Para comprender los actuales desarrollos tecnológicos es necesario remontarse a sus condiciones de posibilidad. La Cuarta Revolución Industrial se caracteriza por la existencia de máquinas y sistemas interconectados en todo el proceso productivo junto a tecnologías digitales y de la automatización como la Inteligencia Artificial, el Internet of Things o el Big Data. ¿Cómo ha sido posible este proyecto?, ¿Cómo hemos llegado hasta él? ¿Qué consecuencias tiene para la consecución de los Objetivos de Desarrollo Sostenible? El viejo ecosistema industrial se esta transformando en un nuevo ecosistema digital. En la fábrica inteligente se aplican nuevos sistemas de comunicación con sistemas ciberfísicos y sensores que facilitan
\end{abstract}

\footnotetext{
${ }^{1}$ Un primer borrador de este trabajo fue presentado el viernes 12 de julio de 2019 en el XIV Congreso de la Asociación Española de Ciencia Política y de la Administración (AECPA) celebrado en Salamanca.
} 
la descentralización y automatización de la toma de decisiones. Al mismo tiempo, la nueva era digital está generando nuevos modelos filosóficos y antropológicos que transforman la visión clásica humanista. El transhumanismo promete potenciar y mejorar la condición humana mediante los avances tecnológicos; isobre qué cosmovisión descansa esta posibilidad? En este nuevo escenario tecnológico, los datos pueden ser utilizados para el control de la conducta, apareciendo formas de vulneración de la privacidad que perfeccionan las ya anunciadas sociedades de control. Esta situación requiere de un replanteamiento de los principios normativos de la nueva era digital. De lo contrario el ODS 5, igualdad de género, o el ODS 16, paz, justicia e instituciones sólidas, no podrán ser alcanzados.

Palabras clave: cuarta revolución industrial; transformación digital; tecnología; Foucault; ODS; derechos humanos.

\title{
The epistemological foundations of the digital transformation and its effects on the 2030 Agenda and human rights
}

\begin{abstract}
To understand the current technological developments it is necessary to go back to their conditions of possibility. The Fourth Industrial Revolution is characterized by the existence of machines and systems interconnected throughout the production process along with digital technologies such as Artificial Intelligence, the Internet of Things or Big Data. How was this project possible? How did we get there? What consequences does it have for the achievement of the Sustainable Development Goals? The old industrial ecosystem is being transformed into a new digital ecosystem. In the intelligent factory new communication systems are applied with cyberphysical systems and sensors that facilitate decentralization and automation of decision making. At the same time, the new digital era is generating new philosophical and anthropological models that transform the classical humanist vision. Transhumanism promises to enhance and improve the human condition through technological advances; On what cosmovision does this possibility rest? In this new technological scenario, data can be used to control behavior, and forms of violation of privacy are appearing, perfecting the already announced control societies. This situation requires a rethinking of the normative principles of the new digital era. Otherwise, SDG 5, gender equality, or SDG 16, peace, justice and solid institutions, cannot be achieved.

Keywords: fourth industrial revolution; digital transformation; technology; Foucault; SDG; human rights.
\end{abstract}

Fecha de recepción: 23/10/2019.

Fecha de aceptación: 23/11/2019. 


\section{OBJETO DE INVESTIGACIÓN}

Los Objetivos de Desarrollo Sostenible (en adelante, ODS) fueron presentados en la Asamblea General de Naciones Unidas el 25 de septiembre de 2015. La Agenda 2030, adoptada por unanimidad por 193 Estados miembros, recoge 17 ODS, 169 metas y 232 indicadores centrados en la persona, el planeta, la prosperidad, la paz y las alianzas, los medios para su implementación y el mecanismo de seguimiento y revisión a escala nacional, regional y global (United Nations General Assembly, 2015). La Agenda 2030 ha sido concebida como un marco universal de gran alcance, una hoja de ruta para la sostenibilidad que está permitiendo ordenar y encauzar el esfuerzo de gobiernos y organizaciones. Los desafíos planteados por los ODS están siendo integrados de manera progresiva en las agendas de administraciones públicas, empresas y tercer sector. Los próximos 10 años han sido definidos como la década de la acción para la consecución de la Agenda 2030.

A pesar del éxito en su adopción y difusión, su estructura conlleva una gran complejidad. El carácter interrelacionado de sus elementos dificulta su implementación, monitorización y medida del desempeño. Además, al analizar su compleja estructura llama la atención el limitado protagonismo que se da a la tecnología, especialmente cuando Klaus Schwab, fundador del Foro Económico Mundial, ha afirmado que estamos ya viviendo el comienzo de la cuarta revolución industrial (Schwab, 2016). Y es que los ODS incorporan la innovación y la tecnología "escondida" en el objetivo 9 y no como el catalizador trasversal de toda la Agenda 2030 (Andreu \& Fernández, 2019).

Desde este punto de vista, la tecnología es la gran oportunidad para acelerar cada uno de los objetivos de la Agenda. La capacidad de innovación de las nuevas tecnologías disruptivas supone algo más que hacer las cosas mejor. La cuarta revolución industrial ofrece un nuevo marco tecnológico que nos acerca a un cambio de paradigma. La convergencia de los mundos físico, digital y biológico que se encuentra en el corazón de la cuarta revolución industrial ofrece oportunidades significativas para que el mundo alcance grandes logros en la eficiencia y el uso de los recursos. El uso de las tecnologías y el diseño de sistemas inteligentes puede minimizar el impacto ambiental, facilitar la transición a una economía circular y recuperar los entornos naturales degradados (Schwab, 2016).

Las tecnologías de la digitalización disuelven el mundo en fragmentos ligeros. La realidad queda simplificada digitalmente. Digital, del latín digitus, viene a significar numérico. El término lo utilizamos para hacer referencia al proceso de traducción de la información en un número (dos cifras 0 y 1 ). El mundo queda reducido a información, que puede descomponerse en unidades: sonidos, colores, imágenes, cantidades, temperaturas. Una máquina podrá registrar esos números y traducirlos 
de nuevo a la información original. Ahora el mundo es almacenable, reproducible y transferible por los ordenadores.

Los procesos de digitalización están generando una inmensa cantidad de datos. Toda esta información -con los nuevos métodos de análisis big data-, ayuda a afrontar diferentes problemas. Las nuevas técnicas pueden medir con mayor precisión los efectos del cambio climático, prevenir los riesgos de inundación o controlar la difusión de enfermedades. El análisis y la toma de decisiones basada en datos puede permitir obtener mejores respuestas y generar planes de acción más eficientes frente a catástrofes futuras.

Sin embargo, es posible que las respuestas basadas en la reducción del mundo a magnitud, el proyecto epistemológico de la modernidad, sean una opción metodológica entre otras posibles. Es necesario preguntarse por el trasfondo epistemológico que nos lleva a una determinada opción metodológica: la de las unidades de medida, la cuantificación y la computación. La tecnología es una condición necesaria, pero, como hipótesis de investigación, insuficiente, para el logro de los ODS. En este sentido podemos formular algunas preguntas que pueden centrar la investigación: ¿en qué lugar queda la ética dentro del conjunto de la Agenda 2030?, ¿de qué ética estamos hablando?, ¿qué consecuencias ambientales (y por tanto humanas, como muestra sistémicamente la Agenda) puede generar en la cuarta revolución industrial?

Respecto a esta última cuestión, llama la atención el carácter paradójico de la tecnología. La concentración de servicios online en centros de datos de gran escala -cada vez más eficientes energéticamente-, ha contribuido a mejorar los niveles de consumo energético, digamos, "por servicio". Pero el aumento constante del tráfico de datos lleva a que se compensen esos incrementos de eficiencia. Y en ello incide el hecho de que la demanda de datos no se limita ya a los servicios digitales, sino que incluye todo tipo de servicios como son los sistemas de monitoreo, vigilancia y control con el auge de sensores y medidores inteligentes (Bellver, 2018, p. 66). ¿Qué consecuencias tiene para los ODS el gasto energético de toda una serie de dispositivos que incluyen ordenadores y smartphones? Según un informe del grupo de investigación The Shift Project (Ferreboeuf, Efoui-Hess \& Kahraman, 2019), si bien la eficiencia energética de las tecnologías digitales ha mejorado significativamente, el uso más amplio de éstas hace que sea cada vez más importante aumentar la concienciación sobre el consumo de energía digital. Los investigadores se refieren a esto como sobriedad digital. ¿Realmente necesitamos un nuevo teléfono móvil cada dos años? La concienciación de los consumidores podría reducir el consumo de energía sin sacrificar ningún avance digital. De lo contrario, las tecnologías digitales podrían ser una amenaza para la consecución de los ODS 7, energía asequible y no contaminante, y, por relación sistémica, para el ODS 13, acción por el clima al aumentar significativamente las emisiones de carbono. 
El ODS 16 llama a promover sociedades más justas, pacíficas e inclusivas. Pero la transformación digital abre una serie de dilemas que podrían limitar la Agenda 2030 (Andreu y Fernández, 2018). Podemos pensar que los algoritmos están diseñados para facilitar la vida de las personas. Pero los algoritmos pueden consolidar desigualdades o aumentar la discriminación hacia determinados grupos minoritarios (Caliskan, Bryson \& Narayanan, 2017). Es necesario preguntarse por el trasfondo ético de los algoritmos y poner en cuestión su posible trasfondo ideológico. Los algoritmos discriminatorios pueden afectar, por ejemplo, al ODS 5 , que busca la igualdad de género y el empoderamiento de mujeres y niñas. Los algoritmos de reconocimiento facial tienen sesgos que han llevado a incriminar a personas racializadas inocentes (Klare, Burge, Klontz, Bruegge \& Jain, 2012). Por otra parte, los riesgos de pérdida de puestos de trabajo por la robotización de labores administrativas o productivas pueden afectar directamente al ODS 8 , que busca trabajo decente y el pleno empleo para todas las personas. Si comparamos las tres empresas más grandes de Detroit en 1990 con las tres empresas más grandes de Silicon Valley en 2014 observamos que generaban una cantidad de ingresos similar, pero con 10 veces menos de empleados en el segundo caso; de 1.2 millones de empleados en Detroit a 137.000 empleados en Silicon Valley (Schwab, 2016, p. 14).

Estamos viviendo el comienzo de una revolución económica y social profunda con importantes consecuencias en la distribución del poder, la riqueza y el conocimiento. Es la mencionada cuarta revolución industrial, que se caracteriza por la existencia de máquinas y sistemas interconectados de forma permanente en todo el proceso productivo. La "Industria 4.0" fue un término acuñado en la Feria de Hannover de 2011 para describir cómo la tecnología revolucionará la organización de las cadenas de valor globales. Mediante la creación de fábricas inteligentes, la cuarta revolución industrial generará un mundo en el que sistemas de fabricación virtuales y físicos cooperarán entre sí de una manera flexible. Esto permite la personalización de los productos y la creación de nuevos modelos de conocimiento del entorno. Tecnologías como la robótica, la inteligencia artificial, el blockchain, el big data, el internet of things, la impresión 3D, los vehículos autónomos, la nanotecnología, la biotecnología o la computación cuántica formarán parte del nuevo ecosistema tecnológico. Para Schwab (2016, pp. 8-9) la cuarta revolución industrial es diferente de la tercera:

1. Por la velocidad, ya que no tiene precedentes históricos y evoluciona a un ritmo exponencial,

2. Por el alcance y la profundidad, no solo se está cambiando el funcionamiento de los procesos, sino que dichos cambios afectan a nuestra subjetividad,

3. Por el impacto en los sistemas, una transformación sistémica que afecta países, industrias y la sociedad en su conjunto. 
A pesar de estos efectos positivos, la posibilidad de una organización cibernéticamente regulada de la sociedad puede hacer realidad la máxima expresión de la mentalidad tecnocrática. Desde un punto de vista epistemológico, esta mentalidad habría sido posible por una comprensión de la razón humana exclusivamente centrada en la comparación lógica de elementos abstractos e iguales, que reduce la realidad a unidades mesurables y computables. Los procesos de objetivación del sujeto pueden alcanzar su máximo esplendor con Revolución 4.0. Los móviles de quinta generación $(5 \mathrm{G})$ y el procesamiento de inteligencia artificial significarán miles de millones de elementos conectados y enormes cantidades de datos en la nube, algo muy valioso para gobiernos y empresas. La ciencia de los datos puede dar lugar a un modelo de toma de decisiones de carácter tecnocrático, minando los procesos democráticos y la autonomía de los sujetos. El artículo 12 de la Declaración Universal de los Derechos Humanos, establece que el derecho a la vida privada es un derecho humano, ya que "nadie será objeto de injerencias arbitrarias en su vida privada, ni su familia, ni cualquier entidad, ni de ataques a su honra o su reputación. Toda persona tiene derecho a la protección de la ley contra tales injerencias o ataques". En consecuencia, los nuevos dispositivos tecnológicos de la sociedad digital dificultarían algunas de las metas del ODS 16, como la meta 16.3 Promover el estado de derecho en los planos nacional e internacional y garantizar la igualdad de acceso a la justicia para todos o la meta 16.10, Garantizar el acceso público a la información y proteger las libertades fundamentales, de conformidad con las leyes nacionales y los acuerdos internacionales.

Estos aspectos obligan a establecer una distinción entre el uso de la tecnología y la ideología tecnocrática. La posición exterior y neutral en la que suele situarse la ideología tecnocrática introduciría sesgos no sometidos a crítica. Es decir, una determinada visión del mundo, de la sociedad, del conocimiento y del ser humano. La producción de subjetividad -a través de los dispositivos móviles con conectividad 5G-, significará un nivel extremo de visualización y monitorización. La filtración de datos, perfiles e imágenes está permitiendo la configuración de precisas bases de datos, facilitando el control de la conducta y los mecanismos de predicción social. Existe el riesgo de desarrollar una economía de mercado basada en los datos, con una nueva y demandada profesión, los data brokers. Este nuevo trabajo consiste en buscar en historiales de compra, publicaciones en redes sociales y otras fuentes disponibles, los datos de personas. A partir de esta recopilación de información, los data brokers construirían perfiles precisos sobre la identidad de los sujetos, que después venderían a anunciantes, agencias de marketing, compañías y organizaciones públicas y privadas, entre otros posibles clientes (Richards y King, 2014). 


\section{LA EPISTEMOLOGÍA DE LA MODERNIDAD}

Este primer apartado trata de responder a la siguiente pregunta: ¿cuáles son los fundamentos epistemológicos que han hecho posible la actual transformación digital? Para responder a esta pregunta, comenzamos describiendo los orígenes del pensamiento moderno. El pensamiento moderno lleva a cabo una simplificación cuantificadora de la realidad a través de la distinción entre cualidades primarias mesurables (extensión, forma, movimiento, cantidad) y secundarias (color, sonido, olor, sabor). El proyecto de la modernidad -tal y como descubrimos en Galileo, Descartes o Hobbes-, apuesta por las cualidades primarias, objetivas, frente a las cualidades subjetivas, producidas por el impacto de la realidad sobre la mente (Nolan, 2011). Descartes, como Galileo, sigue un pensamiento donde sólo conocemos los objetos en términos matemáticos ya que las cualidades secundarias son confusas y no válidas para operaciones matemáticas:

"We know objects only in mathematical terms, and the sole type for him of clear and distinct ideas had come to be mathematical ideas, with the addition of certain logical propositions into which he had been led by the need of a firmer metaphysical basis for his achivements, such as the propositions that we exist, that we think, etc. Hence the secondary qualities, when considered as belonging to the objects, like the primary, inevitably appear to his mind obscure and confused; they are not a clear field for mathematical operations" (Burtt, 1955, p. 118).

El empirismo clásico -y la teoría psicológica fundada sobre él-, habría separado ontológicamente el sujeto de conocimiento y el objeto de conocimiento, relacionándolos epistemológicamente a través de una teoría de la representación (Valor, 2006, p. 180). Para vencer la dificultad de justificar la creencia en la existencia de un mundo exterior a las ideas del sujeto, Locke necesitó desarrollar la teoría del realismo representativo. En ella, las cualidades primarias se corresponderían con la realidad objetiva, mientras que las cualidades secundarias no alcanzarían el estatuto de reales por relacionales:

"Locke thus provided the background of the theory of representative realism. Locke attempted to get over the difficulty of finding a ground, on the basis of his premises, for belief in the existence of a world external to the ideas by drawing a distinction between primary qualities, solidity, size, motion, which are properties of objects, and secondary qualities, like color, sound, odor and pain, which are purely effects of the impact of objective primary properties upon a subject" (Dewey, 1938, pp. 526-527).

El nuevo proyecto científico restringe las cualidades reales de los cuerpos a aquellas que pueden ser comprendidas en términos mecánicos o geométricos, siendo el resto 
de las cualidades meras apariencias derivadas de las primeras. En líneas generales, este proyecto se identifica con los trabajos de Locke y Boyle, pero diferentes versiones de se encuentran en Galileo, Hobbes, Descartes, Spinoza, Newton, Leibniz, Hume o Kant (Nolan, 2011). Las cualidades primarias se corresponderían con el mundo tal y como es en sí mismo. Esta visión sería funcional siempre y cuando no se absolutice, es decir, dentro de sus límites (Naess, 1985, p. 418). Este peligro de absolutización lo estamos viviendo hoy en día.

El desarrollo de la razón de la igualdad lógica (Cassirer, 1972) llevada a cabo en la modernidad fue posible gracias a ciertas reglas metódicas que permitieron la medición y la cuantificación del mundo, separando las ideas claras y distintas de los aspectos subjetivos (secundarios, relacionales y epistemológicamente ficticios). Thomas Hobbes propuso que el razonamiento era como la computación numérica, de forma que nosotros sumamos y restamos silenciosamente en nuestros pensamientos. En el siglo XVII, el matemático y filósofo alemán Gottfried Leibniz afirmó que existía la posibilidad de lograr un lenguaje perfectamente lógico en el que todo el pensamiento se reduciría a cálculos (Russell \& Norvig, 2016). A finales de siglo XIX, Nietzsche juzgará el desarrollo de la modernidad como un incremento, no de la razón, sino de la voluntad de poder, algo que dará lugar a una visión crítica del discurso de la modernidad (Fernández-Mateo, 2014). Esta nueva comprensión del conocimiento pondrá la atención en aquello que ha sido minusvalorado por el pensamiento moderno, declarado como secundario o incluso como una ficción. En su formulación más crítica, serán precisamente esas abstracciones cuantitativas la forma máxima de ficción, que en ningún caso se corresponderían con las cosas tal y como son en sí mismas (Nietzsche, 2010).

A principios de siglo XX, el positivismo lógico o neopositivismo, en su búsqueda del significado, generó un discurso cientifista para establecer un nuevo criterio de demarcación riguroso entre las actividades científicas -con significado o referencia-, y las actividades no científicas, subjetivas o estéticas. Las actividades científicas seguirían un método propio, definido como método de verificación según el positivismo lógico del Círculo de Viena (Ayer, 1959). Karl Popper puso en cuestión ese método (2002, p. 17), proponiendo el falsacionismo como metodología crítica. La postura epistemológica adoptada por Popper fue la de un realismo científico conjetural donde las hipótesis o teorías nunca llegan a superar el estatus de meras conjeturas, lo que señala la falibilidad de la ciencia sin que eso signifique escepticismo o relativismo (Rivadulla, 2015, p. 53). Continuando con el mencionado proyecto de la modernidad -en su vertiente más logicista-, nos encontramos con Rudolf Carnap (1967) y su obra The Logical Structure of the World. Carnap definió un procedimiento computacional explícito para la extracción de conocimiento a partir de experiencias primarias siendo la primera teoría en mostrar la mente como un proceso computacional. Alan Turing, en su famoso artículo Computing 
Machinery and Intelligence (Turing, 1950) sugirió que en vez de preguntar si las máquinas pueden pensar, deberíamos preguntar si las máquinas pueden aprobar un test de inteligencia, conocido como el Test de Turing. En vez de proporcionar una lista larga y quizá controvertida de cualidades necesarias para obtener inteligencia artificialmente, él sugirió una prueba; que el programa mantenga una conversación durante cinco minutos (mediante mensajes escritos en línea) con un interrogador (interlocutor). Éste tiene que averiguar si la conversación se está llevando a cabo con un programa o con una persona; si el programa engaña al interlocutor un 30 por ciento del tiempo, este pasará la prueba.

A pesar del progreso en ciencia y tecnología, la definición del método científico ha sido un problema filosófico de gran envergadura. Si bien es indudable el éxito en la predicción de fenómenos naturales, no parece tan claro que la clave de ese éxito estuviera en la utilización de un método propio (Rorty, 1996, p. 276). Por ello, la cuestión del método científico es objeto de discusión en la actualidad:

"La tradición filosófica que surge a la vez que se desarrolla la ciencia moderna se ha preguntado en qué radica el éxito de esta nueva ciencia, se ha preguntado por las condiciones que han hecho posible una serie de descripciones y predicciones de fenómenos naturales extremadamente precisas. Una de las respuestas ha sido que la clave del éxito estaba en la utilización de un método propio. Éste se ha utilizado a su vez como criterio de distinción entre conocimiento científico y conocimiento no científico. Pero ciertamente no ha habido acuerdo acerca de cuál sea dicho método. Por ello, la cuestión del método ha pasado a ser un problema filosófico de primer orden, y la discusión ha llegado incluso hasta nuestros días" (Valor, 2006, p. 174).

Si analizamos la filosofía de la ciencia contemporánea, el propio neopositivismo de Popper se verá desgastado por los trabajos de Willard Quine, Thomas Kuhn, Imre Lakatos y Paul Feyerabend (Rivadulla, 2015, p. 18). Para Popper, la aparición de un fenómeno que contradiga una teoría debe suponer el rechazo de la teoría, pero para Lakatos la eliminación de los elementos periféricos de una teoría -hipótesis deducidas del núcleo del paradigma-, no implica el cuestionamiento del núcleo del programa de investigación (Lakatos, 1971, p. 99). Ninguna dificultad empírica o anomalía es suficiente para acabar con un paradigma o programa.

Parte de la filosofía de la ciencia contemporánea ha declarado que la existencia de postulados de investigación impide formas de percepción puras. En toda percepción hay una mezcla de observación y teoría. La observación empírica se encuentra filtrada por nuestras ideas previas -un frame-work que hace que la percepción empírica dependa de la teoría. La observación esté cargada de teoría o theory-landen (Hanson, 1958). Lo que percibimos no puede ser independiente de procesos conceptuales, no habiendo conocimiento sin uso del lenguaje (Sellars, 1997). 
La observación está determinada por teorías y éstas están compuestas por postulados de investigación que pueden ser plurales o variables. Como consecuencia de esto, los enunciados de la observación pueden ser heterogéneos. A mediados de siglo XX, la filosofía de la ciencia empieza a hablar, no sólo de la falibilidad de las teorías, sino de la confianza o creencia en los programas de investigación. La crisis de la física clásica dará lugar a una pérdida de confianza en la lógica, que se suma a la pérdida de confianza en la verificación posibilitada por los sentidos por su carga "intrateórica".

El cuestionamiento de los supuestos filosóficos modernos transforma el significado de la objetividad, obteniendo una comprensión distinta del significado de la actividad científica. La objetividad ha sido redefinida como el asentimiento compartido de interpretaciones en virtud de la pertenencia a un mismo paradigma (Kuhn, 1962). El criterio determinante en la admisión de teorías no es la correspondencia teoría-realidad sino el consenso. Al abandonar una referencia metafísica exterior que permita juzgar los diferentes paradigmas, no se puede decir que el paso de un paradigma a otro constituya un progreso en el desarrollo del conocimiento.

¿Qué relación guardan estas ideas con los procesos de digitalización? En la actualidad, el cuestionamiento de la neutralidad de los sistemas de software de las grandes compañías tecnológicas demuestra que no puede hablarse fácilmente de objetividad, abriéndose un debate sobre las diversas formas de sesgo que contienen los algoritmos, un sesgo que descansa en postulados cuya fundamentación última es filosófica (Fernández-Mateo, 2014).

Como vemos, la distinción epistemológica entre aspectos primarios y secundarios ha sido especialmente fructífera hasta el punto de definir en qué consiste ser inteligente. El predominio de los aspectos cuantificables y mesurables -es decir, la reducción del mundo a magnitud (Valor, 2019)-, se ha visto especialmente impulsado por el desarrollo tecnológico posibilitado por la informática, la computación y la digitalización.

Dentro de la informática y las ciencias de la computación, la inteligencia artificial comenzó siendo una disciplina académica que surgió en el entorno de los grandes centros norteamericanos dedicados al estudio de los computadores y del software. Sus primeros éxitos fueron recibidos como extraordinariamente revolucionarios. Pioneros fueron científicos como Herbert Simon, Marvin Minsky, Arthur Samuel o Allen Newell. Lograron éxitos notables con programas que causaban asombro jugando al ajedrez, ejecutando razonamientos lógicos y problemas algebraicos. Pronto fueron considerados como hitos del progreso que anunciaban un futuro de éxitos sorprendentes. La inteligencia artificial nos da soluciones a los problemas, reduce la complejidad del entorno con su búsqueda de patrones, pero siempre en un campo lógico-matemático, porque la inteligencia artificial puede ser vista como ciencia y matemáticas (Simon, 1996). Un algoritmo de búsqueda toma 
como entrada un problema y devuelve como solución una secuencia de acciones. Una vez que encontramos una solución, procedemos a ejecutar las acciones que recomienda (Russell \& Norvig, 2016).

En conclusión, la fundamentación filosófica del éxito científico reside en la transformación que operó en el pensamiento occidental la teoría de las cualidades primarias establecida por los filósofos de la modernidad, y continuada mediante diferentes versiones por los pensadores posteriores. Matemática, lógica y computación han sido hitos progresivos que han definido un determinada y dominante concepción del mundo que reduce el pensamiento a procesos lógicos y matemáticos. Leyendo a Heidegger (2005), podemos preguntar, ison posibles otras formas de pensar?

\section{LAS CONSECUENCIAS SOCIALES DE LA TECNOLOGÍA}

Para justificar el desarrollo de la ciencia y el conocimiento nuevos desarrollos filosóficos se han apoyado en los pensamientos pragmatistas de Charles Pierce, William James y John Dewey (Rivadulla, 2015, p. 18). Un ejemplo es el pensamiento neopragmatista de Richard Rorty, que señala la transformación ético-política de la epistemología y que replantea la división moderna entre lo objetivo y lo subjetivo en términos de consenso público intersubjetivo y estrategias estéticas de índole personal o privado (Rorty, 2008). La convergencia metodológica de autores de matriz pragmatista, como Dewey, y postestructuralista, como Deleuze y Foucault, ha sido puesta de relieve dando lugar a un "parecido de familia" a la hora de definir la noción de problema. Problematización, situación de incertidumbre y plano virtual del pensamiento son conceptos que hacen referencia a un espacio de pensamiento compartido (Fernández-Mateo, 2014).

Estos últimos desarrollos abren la posibilidad de una crítica de los efectos de los artefactos tecnológicos. Una lógica del pensamiento centrada en los aspectos mesurables y computables quizá no sea capaz de dar respuesta a todas las necesidades de las sociedades humanas, generando incluso artefactos fuera de control. En este sentido, este trabajo -sin dejar de reconocer sus virtudes-, es crítico con las posibilidades de un determinado desarrollo tecnológico, definiendo sus límites. Los desarrollos del software no están libres de sesgos raciales y de género (Klare et al., 2012; Caliskan et al., 2017), y la tecnología, supuestamente neutral, esta desarrollando nuevas formas de poder digital y alienación (Helbing et al., 2017). Por eso, gracias a la elección de unas reglas del método distintas es posible establecer una distancia respecto a este tipo de modernidad, definirla como problema y establecer nuevos caminos críticos. El método genealógico de Foucault es una de esas posibilidades. 
El aparato conceptual foucaultiano y su metodología genealógica permite tejer una red de conceptos para describir los procesos de producción y gobierno de la subjetividad en nuestras sociedades digitales. Con Foucault, la comprensión del poder en la sociedad tecnológica no se entiende a partir de la concepción represiva y prohibitiva del poder, el modelo jurídico de la ley. Gracias al cuestionamiento de Foucault, podemos superar el modelo del poder como prohibición -"how is it that our society, Western society in general, has conceived power in such a restricted, isuch a poor and such a negative way?" (Foucault, 2007, p. 154)-, y entenderlo en términos productivos y afirmativos:

"We have not had, until recently, other possibilities of analyzing power besides utilizing these elementary, fundamental, etc., notions that are those of law, of rules, of the sovereign, of the delegation of power, etc. I believe that it is this juridical conception of power, this conception of power derived from law and the sovereign, from rule and prohibition, of which we must now rid ourselves if we want to proceed to an analysis not just of the representation of power, but of the real functioning of power" (Foucault, 2007, p. 156).

La visión afirmativa y productiva del poder comprende la subjetividad como un artefacto susceptible de ser fabricado. Los procesos de subjetivación se articulan mediante las nuevas tecnologías digitales, herramientas cada vez más inmanentes y precisas. Corregir, definir o transformar la identidad del sujeto hoy es más fácil gracias a la capilaridad de las redes sociales. El biopoder (Bazzicalupo, 2016) -el poder sobre la vida de las personas-, fluye a través de las tecnologías de la información y la comunicación (en adelante, TICs) de una forma directa, precisa e instantánea. El paso de una concepción jurídica del poder, concepción negativa, a una visión productiva y afirmativa permite comprender por qué lo que somos puede ser aceptado afirmativamente, es decir, deseado. Lo que hace que el poder sea aceptado es "simply the fact that it doesn't only weigh on us as a force that says no but that it traverses and produces things, it induces pleasure, forms knowledge, produces discourse. It needs to be considered as a productive network which runs through the whole social body" (Foucault, 1980, p. 119). Y es que, en la sociedad digital, el uso de las TICs permite un régimen de observación y control que también produce discursos e induce formas de placer. Es necesario plantear, como problema de investigación, cómo se construye el deseo en la nueva sociedad digital, un deseo que puede llegar a querer su propio control y vigilancia.

A continuación, procedemos a contextualizar brevemente algunas de las nuevas tecnologías presentes en la denominada cuarta revolución industrial: internet of things, big data e inteligencia artificial guiados, principal pero no únicamente, por el aparato conceptual foucaultiano. 


\section{EL INTERNET DE LAS COSAS}

El desarrollo del internet of things nos retrotrae a los orígenes de la informática. En 1948, Norbert Wiener introduce la idea de "cibernética" en su obra Cybernetics or control and communication in the animal and the machine. Wiener perseguía una técnica de control que permitiese automatizar muchos comportamientos de las máquinas sin necesidad de que ese control, en muchísimas fases del proceso, requiriera la intervención humana (Wiener, 1965). Con el término "internet de las cosas" nos referimos a los sistemas que permiten el acceso a la información que poseen y generan multitud de objetos que usamos en la fabricación industrial, la distribución comercial y en la vida cotidiana. La idea de internet de las cosas debe traer a la mente dispositivos wearables, smartphones, realidad aumentada, aplicaciones que están conectadas con el mercado de consumo. Pero su verdadero peso se pronostica en el enriquecimiento de la cadena de valor y, en particular, en el sector industrial. Los datos capturados por sensores permiten ser objeto de estudio mediante muy diversas aplicaciones con técnicas de big data (Andreu \& Fernández, 2018).

Hoy, el internet de las cosas plantea problemas muy diversos, por un lado, en el plano de la seguridad -seguridad de los datos en su fase de producción, gestión, almacenaje y utilización-, y, por otro, en lo que se refiere a la privacidad y uso de los datos -consentimiento y garantía de privacidad-, es decir, cuestiones de carácter ético y político. El uso indebido de los grandes conjuntos de datos personales, recolectados gracias a la tecnología, puede lesionar la privacidad, la reputación e incluso la dignidad de las personas. La producción de datos masivos nos aliena de ellos.

Para dar respuesta a este problema, el Derecho actuaría como límite a la explotación abusiva de las tecnologías: un instrumento regulador de su desarrollo. La garantía de protección de las libertades fundamentales es una de las metas del ODS 16, en particular la meta 16.10 Garantizar el acceso público a la información y proteger las libertades fundamentales, de conformidad con las leyes nacionales y los acuerdos internacionales. Esta meta guarda relación con la Declaración Universal de los Derechos Humanos que establece, en su artículo 12, que Nadie será objeto de injerencias arbitrarias en su vida privada, su familia, su domicilio o su correspondencia, ni de ataques a su honra o a su reputación. Toda persona tiene derecho a la protección de la ley contra tales injerencias o ataques.

El crecimiento de las capacidades (Foucault, 1984, p. 48) desarrollado por las nuevas tecnologías -pensemos en la localización, el tracking posicional y la recopilación de información-, está contribuyendo a reducir crecimiento de la autonomía, al considerar al ciudadano un recurso a explotar. Los trabajos de Foucault han descrito las diversas técnicas mediante las cuales se han transmitido relaciones de poder, generando sujetos con fines económicos. Estas técnicas habrían producido en la 
modernidad sujetos con identidades homogéneas, útiles para su manejo cultural y económico.

Para Foucault cabe preguntarse si la modernidad constituye la continuación de la Ilustración o si es preciso ver ahí una ruptura o una desviación respecto a sus principios fundamentales:

"The relations between the growth of capabilities and the growth of autonomy are not as simple as the eighteenth century may have believed. And we have been able to see what forms of power relation were conveyed by various technologies (whether we are speaking of productions with economic aims, or institutions whose goal is social regulation, or of techniques of communication): disciplines, both collective and individual procedures of normalization exercised in the name of the power of the state, demands of society or of population zones, are examples" (p. 48).

Los datos que capturan los diferentes sensores tecnológicos facilitan una información de altísima utilidad porque retratan con mucha fiabilidad los hábitos que nos constituyen como sujetos (Peirano, 2019). La fábrica de la cultura se expande mediante representaciones, lenguajes, hábitos y prácticas que quedan expresadas en las redes sociales y en diversos sistemas de observación y almacenamiento virtual de la información. El conocimiento de esas circunstancias tiene un enorme valor económico para toda clase de empresas, y también para los poderes públicos. Recopilados de manera centralizada, pueden ser utilizados para el control de la conducta, entrando en formas de biopoder de carácter antidemocrático. La interconexión digital de los objetos cotidianos con internet forma un rizoma digital donde los datos que guardan los dispositivos del internet de las cosas son altamente codiciados debido a que almacenan información sobre la vida de los sujetos. Descubrir la forma de esa nube rizomática, a pesar de los procesos de encriptación, es una amenaza real. $\mathrm{El}$ internet de las cosas se convierte en una tecnología ubicua que hace que los elementos físicos ya no están desconectados del mundo virtual, sino que pueden controlarse de forma remota y pueden actuar como puntos de acceso físico a los servicios de internet (Mattern \& Floerkemeier, 2010).

Si bien el desarrollo del internet of things es una oportunidad para el crecimiento de la economía, también implica riesgos relacionados, no solo con la preservación de los derechos digitales, sino también con el aumento de las emisiones de carbono. Si la procedencia de la energía no es de fuentes renovable incidirá negativamente en el ODS 13, Acción por el Clima. El internet de las cosas requerirá de seis veces más electricidad que el cambio al coche eléctrico, ya que exigirá un 20\% más de electricidad, mientras que el cambio del parque móvil solo supondrá un aumento del 3-4 por ciento de la demanda de electricidad (Efe, 2019). 


\section{BIG DATA}

El big data se presenta como una revolución en la toma de decisiones. La recopilación de cantidades ingentes de datos de diversas fuentes y sistemas de observación requiere de la ayuda de tecnologías informáticas y programas de software capaces de procesarlos. Con el uso del big data, analizar grandes data-sets es mucho más fácil y barato con los nuevos paquetes de software:

"Handling such large datasets has recently become easier through the development of techniques like "Hadoop clusters" which provide a system of shared storage along with "Map Reduce" which provides the analytic layer allowing for reliable and quick access to such large datasets. Facebook reportedly holds its data in a 100-petabyte Hadoop cluster. New computational processing techniques allow for extracting semantic information from data without using an army of human coders and analysts, as would have been required under old techniques" (Tufekci, 2014).

Hoy la vida digital es un objeto de poder: vivimos una era de biopoder digital. La vida digital se ha vuelto un objeto de interés privado. Es necesario llevar más allá los estudios del poder elaborados por Foucault, adentrarlos en nuestra nueva era de la transformación digital. El fundador de Facebook, Mark Zuckerberg, admitió la venta de datos personales de los usuarios a terceros. Y es que, gracias a esta red social, es posible descubrir la identidad de los sujetos sin hacer una sola pregunta. Una gran variedad de atributos personales de las personas -que van desde la orientación sexual hasta la inteligencia-, pueden ser deducidos de forma automática y precisa utilizando sus facebook likes (Kosinski, 2013).

El big data permite perfeccionar la disciplina digital mediante un conocimiento profundo de la vida de las personas. Al poder conocer con precisión los pliegues que constituyen la subjetividad, los hábitos que nos componen, es más fácil corregir su comportamiento hacia pliegues más útiles y convenientes. La disciplina digital, interpretando hoy a Foucault (Foucault, 2007, p. 159) es un proceso de vigilancia, de intensificación del rendimiento, de multiplicación de sus capacidades, de transformación del sujeto en una herramienta útil. Vigilar para conocer, educar para producir un sujeto y corregir sus desviaciones, todo esto es, gracias a la cuarta revolución industrial, más fácil de lo que Foucault pudo pensar.

Hoy, nuestra identidad digital, el sujeto digital que gobierna nuestras prácticas, puede ser la prisión de nuestro cuerpo. La frase mágica es big nudging, la combinación de datos grandes con nudging. Para muchos, esto parece ser una especie de cetro digital que permite gobernar a las masas de manera eficiente, sin tener que involucrar a los ciudadanos en los procesos democráticos: 
"Big data, artificial intelligence, cybernetics and behavioral economics are shaping our society-for better or worse. If such widespread technologies are not compatible with our society's core values, sooner or later they will cause extensive damage. They could lead to an automated society with totalitarian features. In the worst case, a centralized artificial intelligence would control what we know, what we think and how we act. We are at the historic moment, where we have to decide on the right path-a path that allows us all to benefit from the digital revolution" (Helbing et al., 2017).

Si la meta 16.7 plantea Garantizar la adopción en todos los niveles de decisiones inclusivas, participativas y representativas que respondan a las necesidades este aspecto guarda relación directa con el artículo 21 de la Declaración Universal de Derechos Humanos, en particular su artículo 3, La voluntad del pueblo es la base de la autoridad del poder público.

\section{INTELIGENCIA ARTIFICIAL}

El nombre y el origen mismo de la inteligencia artificial como nueva disciplina se debe a John McCarthy, que uso ese término por primera vez en 1956 en un seminario en Darmouth College junto con Marvin Minsky, Nathaniel Rochester, y Claude Shannon (McCarthy, Minsky, Rochester y Shannon, 2006). La inteligencia artificial permite que las máquinas puedan actuar frente a situaciones problemáticas con recursos lógicos, análisis y procesamiento de datos hasta ahora característicos de los humanos. Sin embargo, como se ha descrito al comienzo, esta es una visión del conocimiento exclusivamente centrada en un procedimiento algorítmico mecánico, objetivo y neutral: no se pregunta por quién hace qué para quién y con qué propósitos. En la literatura relacionada con sistemas informáticos, el término bias se ha usado "to refer to computer systems that systematically and unfairly discriminate against certain individuals or groups of individuals in favor of others" (Friedman y Nissenbaum, 1996, p. 332). Hoy es necesario problematizar los procesos hegemónicos inducidos a través de los algoritmos que resuelven los nuevos problemas de la sociedad digital. La tecnología de la automatización promete hacernos la vida más fácil, pero las soluciones que nos ofrece no están exentas de los sesgos que suponen una determinada concepción del mundo.

Para Foucault, el pensamiento "it is what allows one to step back from this way of acting or reacting, to present it to oneself as an object of thought and question it as to its meaning, its conditions, and its goals. Thought is freedom in relation to what one does, the motion by which one detaches oneself from it, establishes it as an object, and reflects on it as a problem" (Foucault, 1984, p. 388). Preguntarse cómo se ha configurado el presente supone salir de ese espacio definido y remontarse a sus condiciones de posibilidad. La problematización es un 
movimiento de distanciamiento respecto de lo que es, un desentendimiento de las formas constituidas al entrar en un espacio de indeterminación. Frente a las respuestas definitivas, Foucault abre un espacio de juego, un claro de libertad para la aparición de nuevas prácticas, de nuevas formas de ser.

Estas prácticas ya esta empezando a ocurrir en el mundo digital. Es el caso de Joy Boulamwini, que, junto con Timnit Gebru han puesto a prueba los sistemas de reconocimiento facial disponibles, llegando a la siguiente conclusión. Cuando los sistemas tienen que identificar a personas racializadas, la tasa de error se dispara, alcanzando, aproximadamente, el 35\% en el caso de las mujeres (Buolamwini \& Gebru, 2018). Boulamwini, fundó la Algorithmic Justice League para denunciar los sesgos raciales y de género lo que ha llevado a que grandes compañías tecnológicas modifiquen sus sistemas de software.

Dentro de las innovaciones en inteligencia artificial, el deep learning permite descubrir patrones de comportamiento que el machine learning no era capaz de extraer; "these methods have dramatically improved the state-of-the-art in speech recognition, visual object recognition, object detection and many other domains such as drug discovery and genomics" (LeCun et al., 2015; Najafabadi et al., 2015). Uno de los grandes hitos del deep learning se produjo en 2012, cuando el equipo de investigación de Google, dirigido por el informático de la Universidad de Stanford Andrew Y. Ng y su compañero de Google Jeff Dean, utilizó una serie de 16.000 procesadores para crear una red neuronal con más de mil millones de conexiones. Luego alimentaron a dicha red neuronal con imágenes aleatorias extraídas de 10 millones de videos de YouTube. El cerebro de Google ensambló una imagen digital de un gato después de haber estado expuesto a millones de imágenes. La máquina de Google aprendió, sin que nadie se lo indicara, su primer concepto abstracto, el de gato, un concepto ideado por ella misma sin mediación humana (Le et al., 2012).

A pesar de esta sorprendente capacidad de resolución de problemas, no todo han sido buenas noticias. Los sesgos culturales y políticos de la inteligencia artificial han quedado demostrados por diversas investigaciones. La inteligencia artificial está siendo un instrumento de discriminación según el género, la raza, la edad o el origen étnico (Greenwald, 2017; Buolamwini \& Gebru, 2018; Kosoff, 2019). La precisión de los algoritmos de detección y clasificación de rostros -los sistemas de reconocimiento facial utilizados por las fuerzas de seguridad estadounidenses-, es sistemáticamente menor para las personas etiquetadas como mujeres, racializadas y entre 18 y 30 años, que para otras cohortes demográficas (Klare et al., 2012). Los estudios de Boulamwini y Gebru (2018) demuestran sustanciales disparidades "in the accuracy of classifying darker females, lighter females, darker males, and lighter males in gender classification systems" lo que ha llevado a empresas tecnológicas como IBM y Microsoft a modificar sus sistemas de software para eliminar los sesgos de sus algoritmos. 
Todos estos sucesos pueden poner en riesgo la consecución de los ODS. La meta 16.b de los ODS plantea Promover y aplicar leyes y políticas no discriminatorias en favor del desarrollo sostenible, algo que conecta de forma directa con el artículo 2 de la Declaración Universal de Derechos Humanos, Toda persona tiene todos los derechos y libertades proclamados en esta Declaración, sin distinción alguna de raza, color, sexo, idioma, religión, opinión política o de cualquier otra índole, origen nacional o social, posición económica, nacimiento o cualquier otra condición.

\section{TECNOLOGÍA Y FUTURO: HUMANISMO, TRANSHUMANISMO Y POSTHUMANISMO}

Por el alcance y la profundidad, la transformación digital y la revolución 4.0 no solo se está cambiando el funcionamiento de los procesos industriales y productivos, sino que dichos cambios afectan a nuestra subjetividad (Schwab, 2016). En este contexto tecnológico, el transhumanismo aparece como el proyecto filosófico que ensalza el crecimiento de las capacidades, un nuevo humanismo que trata de llevar hacia sus límites las capacidades humanas, potenciándolas con la tecnología. El transhumanismo localiza las raíces de su filosofía en el humanismo de la Ilustración. Sin embargo, desde el punto de vista expuesto, detrás de su filosofía está una determinada comprensión de la razón guiada por las cualidades primarias, la lógica de la identidad, los aspectos computacionales y las unidades de medida conmensurables. A partir de estos presupuestos surge una determinada idea de progreso y la esperanza en la creación de un futuro mejor mediante las fuerzas naturales de la razón, la tecnología, el método científico y la creatividad humana.

El humanismo tiende a basarse exclusivamente en el refinamiento educativo y cultural para mejorar la naturaleza humana, mientras que los transhumanistas quieren aplicar la tecnología para superar los límites impuestos por nuestro patrimonio biológico y genético. Para el transhumanismo, la naturaleza humana actual es solo una fotografía fija de un proceso evolutivo que es posible corregir y reorientar. Mediante el uso de la tecnología podemos llegar a transcender lo humano, llegando a ser algo diferente, lo posthumano (More \& Vita-More, 2013, p. 4). Para el transhumanismo, la naturaleza humana perfeccionable "through the use of applied science and other rational methods, which may make it possible to increase human health-span, extend our intelectual and physical capacities, and give us increased control over our own mental states and moods" (Bostrom, 2005, pp. 202-203). Igual que podemos transformar tecnológicamente las organizaciones gracias a la Industria 4.0, aumentando su eficiencia y potencial, así podemos mejorar tecnológicamente la condición humana. Pero la base de este proyecto implica una determinada concepción del mundo; una determinada opción ética, política e incluso una determinada manera de pensar. 
El término singularidad apareció en la conversación de 1958 entre Stanislaw Ulam y John von Neumann, durante la cual hablaron del progreso cada vez más acelerado de la tecnología, de los consiguientes cambios en el modo de vida humana y de la aproximación a una singularidad donde los asuntos humanos, tal y como los conocemos, dejarían de ser los mismos (Ulam, 1958). En 1965, I.J. Good argumentó que el desarrollo de la inteligencia artificial conduciría a una explosión de inteligencia (Good, 1966). Estas ideas fueron retomadas, elaboradas y ampliadas por varios otros escritores influyentes (Bostrom 1998; Kurzweil 1990, 1999; Vinge 1993). Para Kurzweil (2005), el advenimiento de la singularidad nos permitirá transcender nuestras limitaciones biológicas. Podremos vivir cuanto queramos y expandiremos nuestro domino a todo lo que esté a nuestro alcance. La singularidad culminará la fusión de nuestro pensamiento biológico con la tecnología, dando lugar a un mundo que trascenderá nuestras raíces biológicas.

Frente al transhumanismo, los bioconservatives, alarmados por el carácter deshumanizante de las tecnologías, muestran su preocupación que les lleva a pensar "that these technologies might undermine our human dignity or inadvertently erode something that is deeply valuable about being human but that is difficult to put into words or to factor into a cost-benefit analysis" (Bostrom, 2005, p. 203). Este análisis haría necesaria recuperar la antropología trascendental (Fernández-Beites, 2010, Scheler, 2017) que redefina el puesto de las personas en la nueva era digital.

Según sus defensores, el transhumanismo sigue defendiendo el núcleo de las ideas e ideales de la Ilustración (la racionalidad y el método científico, los derechos individuales, la posibilidad y la conveniencia del progreso, la superación de la superstición y el autoritarismo) al tiempo que las revisa y afina a la luz de los nuevos conocimientos. La búsqueda de fundamentos absolutos para la razón, por ejemplo, ha dado paso a una forma más sofisticada, incierta y autocrítica de racionalismo crítico. El yo simple y unificado de la Ilustración ha sido reemplazado por el yo mucho más complejo y desconcertante revelado por las neurociencias. El estatus totalmente único de los seres humanos ha sido sustituido por el entendimiento de que somos parte de un espectro de organismos biológicos y de posibles especies no biológicas del futuro (More \& Vita-More, 2013, p. 10).

Por el contrario, el pensamiento posthumanista, reconociendo las limitaciones filosóficas del humanismo (Braidiotti, 2013) pero sin caer en el antihumanismo (Patton, 1994), plantearía el problema de diferente manera. Las propuestas postestructuralistas han cuestionado algunas de las ideas de la Ilustración, surgiendo un nuevo problema de investigación; distinguir comparativamente las ideas que diferencian el pensamiento humanista, el transhumanista y el posthumanista. Para el pensamiento posthumanista, la afirmación de la racionalidad neutral sin sesgos y la reducción del mundo a magnitud como propuesta metodológica única son ideas cuestionables. A pesar de estas diferencias, pueden identificarse ciertas 
convergencias entre transhumanismo y posthumanismo pues ambos reciben la influencia del pensamiento de Nietzsche (Dosse, 2004; More \& Vita-More, 2013, p. 10).

Desde el punto de vista de los ODS, el interés por la transformación del ser humano a través de la cibernética o la biología sintética es un proceso que puede afectar a la reducción de las desigualdades, el ODS 10. Solo aquellas personas con mayores medios económicos podrían dotarse de una mejor salud o de tecnologías avanzadas de perfeccionamiento humano. Esto conecta con el ODS 3, que limita sus objetivos principalmente a elementos fundamentales de la salud universal de la que a día de hoy carecen todavía miles millones de personas. Estas propuestas podrían desviar recursos para una versión mejorada del sujeto humano que, sin embargo, no tiene sus condiciones ecológicas de existencia garantizadas. No es raro que las propuestas transhumanistas hablen de superar el confinamiento en el planeta Tierra, tal y como establece su Declaración de 2012 (More \& Vita-More, 2013, p. 54). Bajo estos planteamientos, las propuestas transhumanistas darían por terminada la Agenda 2030 en favor de la "agenda" de unos pocos privilegiados.

\section{CONCLUSIÓN}

Para entender los actuales desarrollos tecnológicos es necesario remontarse a los postulados que los han hecho posibles, es decir, a sus condiciones de posibilidad. Estos son los postulados de la corriente principal de la epistemología moderna. La distinción galileana entre cualidades primarias y cualidades secundarias permite separar los elementos cualitativos de la realidad, meras apariencias, de los elementos cuantitativos. Como resultado de esta división, lo objetivo queda reducido a magnitud. La reducción de la realidad a la matemática es sólo una etapa del viaje de la epistemología moderna. El logicismo sostendrá que la matemática es reducible a la lógica, proyecto que inicia Leibniz, en el siglo XVII, pero que será continuado por Frege, en el siglo XIX, y Russell y Whitehead, a principios del siglo XX. Las relaciones lógicas definirían la realidad misma. El proyecto moderno reduciría la realidad a unidades de medida y transitaría de la matemática a la lógica y de la lógica a la computación. En la segunda mitad del siglo XX, los avances en electrónica hicieron posible la construcción del ordenador digital y estas máquinas han permitido la automatización de sistemas formales, superando las barreras que limitaron a Babbage y alcanzando formas de cálculo muy poderosas.

La capacidad de cálculo de las máquinas y sus sistemas de almacenamiento de información están logrando ofrecer diferentes tipos de soluciones a los problemas de la sociedad. Tipos muy distintos de actividades humanas (medicina, transporte, banca) no pueden ser concebidas hoy sin el apoyo que ofrece la informática. Las 
ciencias de la computación aplicadas dominan nuestro mundo, pues tienen la capacidad de resolver problemas del mundo real. Un ejemplo de esto es la inteligencia artificial.

El proyecto moderno implica la aceptación de una determinada cosmovisión, un paradigma epistemológico con sus reglas del método. Por ello, para poder efectuar una crítica de sus artefactos y sus efectos se ha escogido una metodología de análisis distinta, con la que, desde fuera, poder estudiar sus límites. Con estos planteamientos se justifica que el desarrollo de la ciencia y la tecnología no es neutral sino que tiene un origen histórico y no es más que una de las corrientes posibles dentro de la pluralidad del pensamiento humano. Considerar cualquier otra opción metodológica como una mera apariencia sin valor es una forma de absolutismo metodológico sin justificación.

La nueva capacidad de análisis y procesamiento de datos permite descubrir la identidad de los sujetos con una precisión que las técnicas anteriores eran incapaces de extraer. La sociedad de control -gobernada por los datos y sus programas de software big data-, puede dar lugar a nuevas formas de exclusión y privilegio y, por tanto, a nuevas relaciones digitales de poder. Cuestionar nuestro ser histórico digital es el trabajo del pensamiento. Nuestro nuevo ethos digital consiste en una crítica permanente de nuestro ser tecnológico. La identidad personal, como proyecto libre y autónomo, está en peligro por la absolutización de la tecnología, cuyo alcance afecta a millones de personas.

Lo que deja claro esta situación de incertidumbre es que el futuro inmensamente prometedor del internet of things, el big data o la inteligencia artificial puede tener consecuencias muy negativas para las sociedades democráticas. Si esto es así, será difícil alcanzar algunos los ODS que entroncan con los derechos humanos más básicos, pero también con aquellos ODS más medioambientales, por el ingente consumo energético de las nuevas tecnologías si no se corrigen ciertas prácticas.

Los procesos de digitalización tienen implicaciones muy hondas en las formas de creación y control de la opinión pública, y en las relaciones entre la ciudadanía y sus gobernantes. En la era digital, los datos son información, la información es poder. El uso de las redes sociales deja inevitablemente trazas digitales de gran valor para empresas y organizaciones. Esta información es muy útil para organizaciones públicas y privadas, que pueden hacer un uso no democrático de ella. Por otra parte, es necesaria una reflexión antropológica sobre el ser humano que pueda introducir una visión no simplemente cuantitativa; aquella que define el pensamiento como una lógica abstracta que computa igualdades. Queda para un futuro análisis el estudio del actual capitalismo tecnológico y sus líderes, los denominados GAFA (Google, Amazon, Facebook y Apple). 


\section{BIBLIOGRAFÍA}

Andreu, A. \& Fernández, J. (2018). El propósito de los datos: introducción al Data Ethics. En Aspectos legales, retos y reflexiones para el desarrollo de la responsabilidad social: memoria académica curso 2017-2018 (pp. 113-126). Madrid: Universidad Pontificia Comillas.

Andreu, A. \& Fernández, J. (2019, 17 de Septiembre). La ambivalencia tecnológica para impulsar (¿o no?) los ODS. Telos. Cuadernos de comunicación, tecnología y sociedad, 111. Recuperado de: https://telos.fundaciontelefonica.com/ambivalencia-tecnologica-para-impulsar-ods-onu/

Ayer, A. J. (1959). Logical positivism. New York: Simon and Schuster.

Bazzicalupo, L. (2016). Biopolítica: un mapa conceptual. Madrid: Melusina.

Bellver, J. (2018). Costes y restriciones ecológicas al capitalismo digital. Papeles de relaciones ecosociales y cambio global, 144, 59-77.

Bostrom, N. (1998). How Long Before Superintelligence? International Journal of Futures Studies, 2. Recuperado de: https://www.nickbostrom.com/superintelligence.html

Bostrom, N. (2005). In defense of posthuman dignity. Bioethics, 19 (3), 202-214. https://doi.org/10.1111/j.1467-8519.2005.00437.x

Braidotti, R. (2013). The posthuman. Cambridge, UK: Polity Press.

Buolamwini, J. \& Gebru, T. (2018). Gender shades: Intersectional accuracy disparities in commercial gender classification. En Conference on Fairness, Accountability and Transparency (pp. 77-91). Recuperado de: http://proceedings.mlr.press/v81/ buolamwini18a/buolamwini18a.pdf

Burtt, E. A. (2003). The metaphysical foundations of modern physical science: A historical and critical essay. New York: Dover publications

Caliskan, A., Bryson, J. J., \& Narayanan, A. (2017). Semantics derived automatically from language corpora contain human-like biases. Science, 356 (6334), 183-186. https://doi.org/10.1126/science.aal4230

Carnap, R. (1967). The logical structure of the world. Berkeley y Los Angeles: University of California Press.

Cassirer, E. (2008). La filosofía de la Ilustración. Madrid: Fondo de Cultura Económica.

Churchland, P. M. (2013). Matter and consciousness. Cambridge, Massachusetts: MIT press.

Dewey, J. (1938). Logic. The Theory of Inquiry. New York: Henry Holt \& Co.

Dosse, F. (2004). Historia del estructuralismo, Tomo II: El canto del cisne, 1967 hasta nuestros días. Madrid: Ediciones Akal.

Efe (2019) Internet de las cosas requerirá 6 veces más energía que el coche eléctrico. Eldiario.es. Recuperado de: https://www.eldiario.es/economia/Internet-cosas-requerira-energia-electrico 0 872813105.html 
Fernández-Beites, P. (2010). Tiempo y sujeto: después de Heidegger. Madrid: Encuentro. Fernández-Mateo, J. (2014). El nuevo sujeto estético en la sociedad de la información: una genealogía de los procesos de subjetivación. Tesis Doctoral. Universidad Complutense de Madrid.

Ferreboeuf, H., Efoui-Hess, M. \& Kahraman, Z. (2019). Lean ICT: towards digital sobriety The Shift Project. Recuperado de: https://theshiftproject.org/wp-content/ uploads/2019/03/Lean-ICT-Report The-Shift-Project 2019.pdf

Foucault, M. (1980). Power/knowledge: Selected interviews and other writings, 1972 1977. New York: Pantheon.

Foucault, M. (1984). The Foucault Reader. New York: Pantheon.

Foucault, M. (2007). The meshes of power. En Crampton, J. W. \& Elden, S. (eds.), Space, Knowledge and Power: Foucault and Geography. Aldershot: Ashgate.

Friedman, B., \& Nissenbaum, H. (1996). Bias in computer systems. ACM Transactions on Information Systems (TOIS), 14 (3), 330-347. https://doi. org $/ 10.1145 / 230538.230561$

Greenwald, A. G. (2017). An AI stereotype catcher. Science, 356 (6334), 133-134. https://doi.org/10.1126/science.aan0649

Good, I. J. (1966). Speculations concerning the first ultraintelligent machine. En Advances in computers (vol. 6, pp. 31-88). Elsevier.https://doi.org/10.1016/ s0065-2458(08)60418-0

Hanson, N. R. (1958). Patterns of Discovery: An Inquiry into the Conceptual Foundations of Science. Cambridge: Cambridge University Press.

Heidegger, M. (2005). ¿Qué significa pensar? Madrid: Trotta.

Helbing, D., Frey, B. S., Gigerenzer, G., Hafen, E., Hagner, M., Hofstetter, Y. \& Zwitter, A. (2017). Will Democracy Survive Big Data and Artificial Intelligence? We are in the middle of a technological upheaval that will transform the way society is organized. We must make the right decisions now. Scientific American. Recuperado de: https://www.scientificamerican.com/article/will-democracy-survive-big-data-and-artificial-intelligence/

Klare, B. F., Burge, M. J., Klontz, J. C., Bruegge, R. W. V., \& Jain, A. K. (2012). Face recognition performance: Role of demographic information. IEEE Transactions on Information Forensics and Security, 7 (6), 1789-1801. https://doi.org/10.1109/ tifs.2012.2214212

Kosinski, M., Stillwell, D. \& Graepel, T. (2013). Private traits and attributes are predictable from digital records of human behavior. Proceedings of the National Academy of Sciences, 110 (15), 5802-5805. https://doi.org/10.1073/pnas.1218772110 Kosoff, M. (2019). Alexandria Ocasio-Cortez Says Algorithms Can Be Racist. Here's Why She's Right. www.livesciencie.com. Recuperado de: https://www. livescience.com/64621-how-algorithms-can-be-racist.html 
Kuhn, T. S. (1962). The structure of scientific revolutions. Chicago: University of Chicago Press.

Kurzweil, R. (1990). The Age of Intelligent Machines. Cambridge, MA: MIT Press.

Kurzweil, R. (1999). The Age of Spiritual Machines: When Computers Exceed Human Intelligence. New York: Viking.

Kurzweil, R. (2005). The singularity is near. London: Gerald Duckworth \& Co.

Lakatos I. (1971). History of Science and its Rational Reconstructions. En Buck R.C., Cohen R.S. (eds.) PSA 1970. Boston Studies in the Philosophy of Science, vol 8. Springer, Dordrecht. https://doi.org/10.1007/978-94-010-3142-4 7

Le, Q. V., Ranzato, M. A., Monga, R., Devin, M., Chen, K., Corrado, G. S., ... \& $\mathrm{Ng}, \mathrm{A}$. Y. (2012). Building high-level features using large scale unsupervised learning. En Proceedings of the 29th International Conference on International Conference on Machine Learning (pp. 507-514). Edinburgh, Scotland, UK: Omnipress. https://doi.org/10.1109/icassp.2013.6639343

LeCun, Y., Bengio, Y., \& Hinton, G. (2015). Deep learning. Nature, 521 (7553), 436-444. https://doi.org/10.1038/nature14539

Mattern, F., \& Floerkemeier, C. (2010). From the Internet of Computers to the Internet of Things. En From active data management to event-based systems and more (pp. 242-259). Berlin, Heidelberg: Springer. https://doi.org/10.1007/9783-642-17226-7 15

McCarthy, J., Minsky, M. L., Rochester, N., \& Shannon, C. E. (2006). A proposal for the dartmouth summer research project on artificial intelligence, august 31, 1955. AI magazine, 27 (4), 12. https://doi.org/10.1609/aimag.v27i4.1904

More, M., \& Vita-More, N. (eds.). (2013). The transhumanist reader: Classical and contemporary essays on the science, technology, and philosophy of the human future. United Kingdom: John Wiley \& Sons.

Naess, A. (1985). The world of concrete contents. Inquiry, 28 (1-4), 417-428. https://doi.org/10.1080/00201748508602059

Najafabadi, M. M., Villanustre, F., Khoshgoftaar, T. M., Seliya, N., Wald, R., \& Muharemagic, E. (2015). Deep learning applications and challenges in big data analytics. Journal of Big Data, 2 (1), 1. https://doi.org/10.1186/s40537-014-0007-7

Nietzsche, F. W. (2010). Sobre verdad y mentira en sentido extramoral y otros fragmentos de filosofía del conocimiento. Madrid: Tecnos.

Nolan, L. (ed.). (2011). Primary and secondary qualities: The historical and ongoing debate. Oxford, UK: Oxford University Press.

Patton, P. (1994). Foucault's subject of power. Political Theory Newsletter, 6 (1), 60-71.

Peirano, M. (2019). El enemigo conoce el sistema. Barcelona: Debate.

Popper, K. (2002). The logic of scientific discovery. London-New York: Routledge. 
Richards, N. M., \& King, J. H. (2014). Big data ethics. Wake Forest L. Review, 49, 393. Recuperado de: https://heinonline.org/HOL/LandingPage?handle=hein. journals $/$ wflr 49 \&div $=16 \&$ id $=$ \&page $=$

Rivadulla, A. (2015). Meta, método y mito en ciencia. Madrid: Editorial Trotta.

Rorty, R. (1996). Consecuencias del pragmatismo. Madrid: Tecnos.

Rorty, R. (2008): Filosofía y Futuro. Barcelona: Gedisa Editorial.

Russell, S. J. \& Norvig, P. (2016). Artificial intelligence: a modern approach. Malaysia: Pearson Education Limited.

Scheler, M. (2017). El puesto del hombre en el Cosmos. Madrid: Escolar y Mayo Editores.

Sellars, W. (1997). Empiricism and the Philosophy of Mind. Cambridge: Harvard University Press.

Simon, H. A. (1996). The sciences of the artificial. Cambridge, Massachusetts: MIT press.

Schwab, K. (2016). The Fourth Industrial Revolution. Cologny/Geneva, Switzerland: World Economic Forum.

Tufekci, Z. (2014). Engineering the public: Big data, surveillance and computational politics. First Monday, 19 (7). https://doi.org/10.5210/fm.v19i7.4901

Turing, A. M. (1950). Computing machinery and intelligence. Mind, 59 (236), 433-460. https://doi.org/10.1093/mind/lix.236.433

Ulam, S. (1958). John von Neumann 1903-1957. Bulletin of the American Mathematical Society, 64 (3), 1-49. https://doi.org/10.1090/s0002-9904-1958-10189-5

United Nations General Assembly (2015). Transforming our world: The 2030 agenda for sustainable development. Retreived from: http://www.un.org/ga/search/ view doc.asp?symbol=A/RES/70/1\&Lang $=\mathrm{E}$

United Nations General Assembly (1948). Universal declaration of human rights. United Nations General Assembly. Recuperado de: https://www.un.org/en/ universal-declaration-human-rights/

Valor, J. A. (2006). Utilidad y objetividad en la investigación científica. Revista de Filosofía, 31 (2), 173-188. Recuperado de: https://revistas.ucm.es/index.php/ RESF/article/view/RESF0606220173A/9317

Valor, J. A. (13 de mayo 2019). Inteligencia artificial e inteligencia humana: ¿competencia u oportunidad de colaboración? Encuentro Ciencia y Filosofía: ¿Es posible que la Inteligencia Artificial genere Teorías Científicas? Madrid: Real Academia de Ciencias Exactas, Físicas y Naturales.

Vernor, V. (1993). The coming technological singularity. Whole Earth Review, Winter. Wiener, N. (1965). Cybernetics or control and communication in the animal and the machine. Cambridge, Massachusetts: MIT press. 